Copyright by the Acoustical Society of America. Cramer, M. S. \& Andrews, M. F. (2003). A modified Khokhlov-Zabolotskaya equation governing

shear waves in a prestrained hyperelastic solid. Journal of the Acoustical Society of America, 114(4), 1821-1832. doi: 10.1121/1.1610460

\title{
A modified Khokhlov-Zabolotskaya equation governing shear waves in a prestrained hyperelastic solid
}

\author{
M. S. Cramer and M. F. Andrews \\ Department of Engineering Science and Mechanics, Virginia Polytechnic Institute \& State University, \\ Blacksburg, Virginia 24061-0219
}

(Received 27 January 2003; revised 15 July 2003; accepted 28 July 2003)

\begin{abstract}
Weakly nonlinear, weakly diffracting, two-dimensional shear waves propagating in a prestrained hyperelastic solid are examined. A modification of the classical Khokhlov-Zabolotskaya equation is derived using a systematic perturbation scheme. Both dissipative and nondissipative materials were considered. The principal effect of the prestrain was seen to be the inclusion of a quadratic nonlinearity to the cubic nonlinearity found in the case of zero prestrain. Further new results include the shock jump relations and the prediction of shocks having a speed which is identical to the nonlinear wave speed ahead of or behind the shock. Explicit expressions for the nonlinearity coefficients for the special case of a Blatz-Ko material were provided. (C) 2003 Acoustical Society of America. [DOI: 10.1121/1.1610460]
\end{abstract}

PACS numbers: 43.28.Kt, 43.28.Lv, 43.28.Js [MFH]

\section{INTRODUCTION}

The canonical equation for weakly nonlinear and weakly diffracting waves is the Khokhlov-Zabolotskaya (KZ) equation, which can be written in the general form

$$
\left(U_{\tau}+\beta^{\prime} U U_{\chi}\right)_{\chi}+\frac{1}{2} U_{\eta \eta}=0,
$$

where $U=U(\chi, \eta, \tau)$ is a measure of the wave disturbance, $\chi$ is a spatial variable measured in a frame moving with the wave, $\eta$ is a transverse spatial variable, and $\tau$ is a time-like variable. The order-one constant $\beta^{\prime}$ is a measure of the nonlinearity of the system. The $\mathrm{KZ}$ equation is recognized as being quadratically nonlinear, and we therefore refer to $\beta^{\prime}$ and similar parameters as quadratic nonlinearity parameters. The first derivation of this equation was given by Zabolotskaya and Khokhlov (1969) in the context of ordinary nonlinear acoustics.

When modified to account for dispersion and dissipation, the $\mathrm{KZ}$ equation (1) provides an accurate description of many commonly encountered physical systems including those associated with the nonlinear acoustics of low-pressure gases and liquids, the nonlinear acoustics of longitudinal waves in solids, magnetosonic and acoustic waves in magnetohydrodynamics, and surface and internal waves in the ocean. However, in many systems of practical interest, the quadratic nonlinearity coefficient $\beta^{\prime}$ may be small or vanish altogether. In such cases, (1) becomes

$$
U_{\tau \chi}+\frac{1}{2} U_{\eta \eta} \approx 0,
$$

and nonlinearity no longer plays a role in the wave evolution, at least over the length and time scales originally associated with (1). Examples where $\beta^{\prime}$ is identically zero are nonlinear optics, Alfvén waves in magnetohydrodynamics, and shear waves in an isotropic nonlinear solid; it is the latter application which is of central interest in the present paper.

While (2) may give a reasonable description of the wave evolution in some applications, any complete understanding of nonlinear diffraction in systems having $\beta^{\prime}=0$ will require further study. It may also be shown that waves governed by
(2) may focus if the initial wavefront is curved. Near the focal region (2) must be modified to include nonlinear effects; the resultant evolution near the focal region is also expected to be governed by a nonlinear equation of the $\mathrm{KZ}$ type.

The evolution equation governing systems having small or zero $\beta^{\prime}$ will necessarily involve an inclusion and analysis of cubically nonlinear terms in addition to the quadratically nonlinear terms normally leading to (1). Zabolotskaya (1986) has derived the explicit form of the extension of (1) for the case of shear waves propagating in a nonlinear solid having no strain in the undisturbed state. The result of Zabolotskaya's study was that the extension of (1) for shear waves can be written as

$$
\left(U_{\tau}+\beta^{\prime \prime} U^{2} U_{\chi}\right)_{\chi}+\frac{1}{2} U_{\eta \eta}=0,
$$

at least when the displacements are confined to the $\chi-\eta$ plane and dissipation is ignored. Here, $\beta^{\prime \prime}$ is a cubic nonlinearity coefficient which depends on the particular material model. Zabolotskaya's result (3) is intuitively satisfying because the net effect of the detailed perturbation analysis carried out by Zabolotskaya is to replace the $\beta^{\prime} U U_{\chi}$ term in (1) by the cubic term $\beta^{\prime \prime} U^{2} U_{\chi}$ when $\beta^{\prime}=0$. Under the conditions implicit in Zabolotskaya's derivation it can be shown that the parameter $\beta^{\prime \prime}$ can be computed by an analysis of the one-dimensional problem, i.e., a problem where all variations with respect to the transverse direction vanish, thus suggesting a simplified derivation or a check on the value of $\beta^{\prime \prime}$. Of course, similar remarks can be made for the coefficient $\beta^{\prime}$ seen in (1).

More general studies of cubically nonlinear systems have been carried out by Kluwick and Cox (1998) and Cramer and Webb (1998). The first set of authors developed a general multiple-scales scheme for the treatment of cubically nonlinear systems, i.e., systems in which the relevant $\beta^{\prime}$ is either zero or small. Kluwick and Cox's results included those for ordinary nonlinear acoustics on rays as well as the extension for diffracting systems. Cramer and Webb 
(1998) presented a more restricted version of Kluwick and Cox's scheme focused on nonlinear diffraction. The primary extension of Cramer and Webb's work over that of Kluwick and Cox was the inclusion of the effects of dispersion, dissipation, and relaxation.

A remarkable feature of the general schemes presented by Kluwick and Cox (1998) and Cramer and Webb (1998) is that the general form of the $\mathrm{KZ}$ equation valid for small or zero $\beta^{\prime}$ is not of the form (3), but must be supplemented with quadratic terms representing an interaction of diffraction and nonlinearity. In the notation used for Eqs. (1)-(3), the terms which must be added to (3) can be written

$$
\left\{\beta^{\prime \prime \prime} U U_{\eta}+\beta^{(i v)} U_{\chi} U_{\eta} d \chi\right\}_{\chi},
$$

where $\beta^{\prime \prime \prime}$ and $\beta^{(i v)}$ are system-dependent constants. Both $U_{\eta}$ and $\int U_{\eta} d \chi$ can be shown to be zero when diffraction is negligible.

For a general system, there is no a priori reason why the material constants $\beta^{\prime \prime \prime}$ and $\beta^{(i v)}$ should vanish. However, previous investigations have shown that $\beta^{\prime \prime \prime}$ and $\beta^{(i v)}$ do in fact vanish for many cases of practical interest; an example of direct interest here is the case studied by Zabolotskaya (1986). Andrews (1999) has shown that these coefficients also vanish for the case of nonlinear optics. Kluwick and Cox (1998) and Cramer and Webb (1998) have shown that $\beta^{\prime \prime \prime}$ and $\beta^{(i v)}$ are zero for the case of acoustic waves in a Bethe-Zel'dovich-Thompson fluid; the latter class of fluid has a quadratic nonlinearity parameter $\beta^{\prime}$ which can be small or even change sign at certain thermodynamic states. However, Cramer and Webb (1998) have also shown that the coefficients $\beta^{\prime \prime \prime}$ and $\beta^{(i v)}$ are nonzero for nonlinear Alfvén waves in a plasma. Thus, although the general form of the $\mathrm{KZ}$ equation for cubically nonlinear problems is known from the work of Kluwick and Cox (1998) and Cramer and Webb (1998), the status of the additional terms proportional to $\beta^{\prime \prime \prime}$ and $\beta^{(i v)}$ will depend on the details of the specific application. Furthermore, the values of $\beta^{\prime \prime \prime}$ and $\beta^{(i v)}$ cannot be determined by or carried over from a simpler case, e.g., by an analysis of the one-dimensional theory.

In the present investigation we examine the effect of a weak prestrain on the nonlinear diffraction of shear waves. In particular, we will determine the modification to the cubic $\mathrm{KZ}$ equation, e.g., (3), required when the undisturbed state has a small shear strain. Such prestrained states can be of interest in acoustoelasticity (Norris, 1998) and may also occur naturally. In the present study we show that the effect of the prestrain will be to modify the linear wave speed and to introduce nonvanishing quadratic nonlinearity in the final $\mathrm{KZ}$ equation. A further question of interest is whether the prestrain will result in terms similar to those proportional to $\beta^{\prime \prime}$, $\beta^{(i v)}$ which are nonzero. We also account for the shock waves which will inevitably form and record the shock speed for diffracting shear waves in a prestrained material. A final extension of Zabolotskaya's results is to determine the form of the nonlinearity constants for the case of a specific material model; this model is based on the well-known BlatzKo strain energy.

\section{FORMULATION}

The stress-strain relation for a general hyperelastic solid undergoing isothermal deformations is

$$
\sigma_{i j}=\alpha_{0} \delta_{i j}+\alpha_{1} B_{i j}+\alpha_{2} B_{i m} B_{m j},
$$

where $\sigma_{i j}$ is the Cauchy stress tensor, $\delta_{i j}$ is the Kronecker delta, and $B_{i j}$ is the left Cauchy-Green tensor. In all that follows, lower-case Latin indices will range over 1, 2, 3 and the Einstein summation convention will be employed. The left Cauchy-Green tensor is defined as

$$
B_{i j} \equiv F_{i \ell} F_{j \ell}=B_{j i},
$$

where

$$
F_{i j} \equiv \frac{\partial x_{i}}{\partial X_{j}}
$$

is the deformation matrix, $X_{i}$ are the Cartesian coordinates of a material particle in the undeformed (natural) configuration, and $x_{i}$ are the coordinates of its position in the deformed configuration. We will refer to $X_{i}$ as the Lagrangian coordinates and $x_{i}$ as the Eulerian coordinates.

The scalar coefficients $\alpha_{0}, \alpha_{1}, \alpha_{2}$ are related to the strain energy per unit mass by

$$
\begin{aligned}
& \alpha_{0}=2 \rho_{0} I I I^{1 / 2} \frac{\partial U}{\partial I I}, \\
& \alpha_{1}=\frac{2 \rho_{0}}{I I I^{1 / 2}}\left(\frac{\partial U}{\partial I}+I \frac{\partial U}{\partial I I}\right), \\
& \alpha_{2}=-\frac{2 \rho_{0}}{I I I^{1 / 2}} \frac{\partial U}{\partial I I},
\end{aligned}
$$

where $U \equiv U(I, I I, I I I)$ is the aforementioned strain energy and the constant $\rho_{0}$ is the material density in the unstrained state. The quantities $I, I I, I I I$ are the invariants of $B_{i j}$ given by

$$
\begin{aligned}
& I \equiv B_{\ell \ell}, \quad I I \equiv \frac{1}{2}\left(I^{2}-B_{i m} B_{m i}\right), \\
& I I I \equiv \operatorname{det}\left(B_{i j}\right),
\end{aligned}
$$

where $\operatorname{det}\left(B_{i j}\right)$ refers to the determinant of $B_{i j}$.

Motions of the solid are governed by Cauchy's equation

$$
\rho \ddot{u}_{i}=\frac{\partial \sigma_{j i}}{\partial x_{j}},
$$

where the dots denote differentiation with respect to time following the particle, $u_{i}\left(X_{\ell}, t\right)$ is the displacement vector defined by

$$
u_{i} \equiv x_{i}-X_{i},
$$

and $\rho$ is the instantaneous material density related to the density in the unstrained configuration $\left(\rho_{0}\right)$ by

$$
\rho=\rho_{0} I I I^{-1 / 2}=\rho_{0} F^{-1},
$$

where $F \equiv \operatorname{det}\left(F_{i j}\right)$. A useful alternate form of (12) is obtained by recasting it in terms of the Lagrangian coordinates $X_{i}$ as follows: 


$$
\rho_{0} \frac{\partial^{2} u_{i}}{\partial t^{2}}=F \frac{\partial X_{p}}{\partial x_{j}} \frac{\partial \sigma_{j i}}{\partial X_{p}},
$$

where the time differentials are taken with $X_{i}$ held fixed.

At this stage we note that the exact relation (5) can be reduced to the usual linear stress-strain relation by taking $u_{i}$ to be small and retaining only terms linear in $\partial u_{i} / \partial X_{j}$. The resultant linearized form of (5) is found to be

$$
\sigma_{i j} \approx \lambda^{\prime} \frac{\partial u_{\ell}}{\partial X_{\ell}} \delta_{i j}+\mu^{\prime}\left(\frac{\partial u_{i}}{\partial X_{j}}+\frac{\partial u_{j}}{\partial X_{i}}\right),
$$

where

$$
\left.\mu^{\prime} \equiv \tilde{\alpha}_{1}\right|_{0}, \quad \lambda^{\prime} \equiv 2 \widetilde{\beta}_{0},
$$

are the usual Lamé constants, and we have required that

$$
\tilde{\alpha}_{0}=\tilde{\alpha}_{0}(I, I I, I I I) \equiv \alpha_{1}+\alpha_{2}+\alpha_{3}
$$

vanishes at the undeformed state $I=I I=3, I I I=1$; the latter condition ensures that the stress vanishes when the strain does. In the remainder of this paper we will find it convenient to define the following quantities:

$$
\begin{aligned}
& \widetilde{\alpha}_{1} \equiv \alpha_{1}+2 \alpha_{2}, \\
& \tilde{\alpha}_{2} \equiv \alpha_{2},
\end{aligned}
$$

and

$$
\left.\tilde{\beta}_{0} \equiv \frac{\partial \tilde{\alpha}_{0}}{\partial I}\right|_{0}+\left.2 \frac{\partial \widetilde{\alpha}_{0}}{\partial I I}\right|_{0}+\left.\frac{\partial \widetilde{\alpha}_{0}}{\partial I I I}\right|_{0},
$$

where the notation $\left.()\right|_{0}$ will denote quantities evaluated at the unstrained state $I=I I=3, I I I=1$.

The perturbations to the undisturbed state will be taken to be two-dimensional, i.e., $u_{i}=u_{i}\left(X_{1}, X_{2}, t\right)$ only and

$$
\begin{aligned}
& x_{1}=X_{1}+u_{1}\left(X_{1}, X_{2}, t\right), \\
& x_{2}=X_{2}+u_{2}\left(X_{1}, X_{2}, t\right), \\
& x_{3}=X_{3} .
\end{aligned}
$$

Under these conditions, the left Cauchy-Green tensor (6) can be written

$$
\begin{aligned}
& B_{11}=1+2 e_{11}+\left(e_{11}\right)^{2}+\left(e_{12}\right)^{2}, \\
& B_{12}=B_{21}=\left(1+e_{11}\right) e_{21}+\left(1+e_{22}\right) e_{12}, \\
& B_{22}=1+2 e_{22}+\left(e_{22}\right)^{2}+\left(e_{21}\right)^{2}, \\
& B_{33}=1,
\end{aligned}
$$

with all other components equal to zero. Here

$$
e_{i j} \equiv F_{i j}-\delta_{i j}=\frac{\partial u_{i}}{\partial X_{j}} \neq e_{j i}
$$

represents the deviation of the deformation matrix from the undisturbed state and should not be confused with the strain; in the linear approximation, the latter can be written as $\left(e_{i j}\right.$ $\left.+e_{j i}\right) / 2$. The invariants (11) now reduce to

$$
\begin{aligned}
& I=1+B_{11}+B_{22}, \\
& I I=B_{11}+B_{22}+B_{11} B_{22}-B_{12}^{2},
\end{aligned}
$$

$$
I I I=B_{11} B_{22}-B_{12}^{2},
$$

with $B_{i j}$ given by (22). When (22) is substituted in (5), we find that the only nonzero components of the stress are

$$
\begin{aligned}
\sigma_{11}= & \tilde{\alpha}_{0}+\tilde{\alpha}_{1}\left(2 e_{11}+e_{11}^{2}+e_{12}^{2}\right) \\
& +\tilde{\alpha}_{2}\left(Q_{112}+Q_{113}+Q_{114}\right), \\
\sigma_{22}= & \tilde{\alpha}_{0}+\tilde{\alpha}_{1}\left(2 e_{22}+e_{22}^{2}+e_{21}^{2}\right) \\
& +\tilde{\alpha}_{2}\left(Q_{222}+Q_{223}+Q_{224}\right), \\
\sigma_{12}= & \sigma_{21}=\tilde{\alpha}_{1}\left(e_{21}+e_{12}+e_{11} e_{21}+e_{22} e_{12}\right) \\
& +\tilde{\alpha}_{2}\left(Q_{122}+Q_{123}+Q_{124}\right), \\
\sigma_{33}= & \tilde{\alpha}_{0},
\end{aligned}
$$

where

$$
\begin{aligned}
Q_{112} \equiv & 4 e_{11}^{2}+e_{21}^{2}+2 e_{21} e_{12}+e_{12}^{2} \\
Q_{113} \equiv & 4 e_{11}^{3}+4 e_{11} e_{12}^{2}+2 e_{11} e_{21}^{2}+2\left(e_{11}+e_{22}\right) e_{21} e_{12} \\
& +2 e_{22} e_{12}^{2} \\
Q_{114} \equiv & e_{11}^{4}+2 e_{11}^{2} e_{12}^{2}+e_{12}^{4}+e_{11}^{2} e_{21}^{2}+2 e_{22} e_{11} e_{21} e_{12} \\
& +e_{22}^{2} e_{12}^{2} \\
Q_{222}= & 4 e_{22}^{2}+e_{21}^{2}+2 e_{21} e_{12}+e_{12}^{2} \\
Q_{223}= & 4 e_{22}^{3}+2 e_{11} e_{21}^{2}+2 e_{11} e_{21} e_{12}+2 e_{22} e_{21} e_{12}+2 e_{22} e_{12}^{2} \\
& +4 e_{22} e_{21}^{2}, \\
Q_{224}= & e_{22}^{4}+2 e_{22}^{2} e_{21}^{2}+e_{21}^{4}+e_{11}^{2} e_{21}^{2}+2 e_{22} e_{11} e_{12} e_{21} \\
& +e_{22}^{2} e_{12}^{2} \\
Q_{122} \equiv & 2\left(e_{11}+e_{22}\right)\left(e_{21}+e_{12}\right) \\
Q_{123} \equiv & \left(e_{21}+e_{12}\right)\left(e_{12}^{2}+2 e_{11} e_{22}+e_{21}^{2}\right)+3 e_{11}^{2} e_{21} \\
& +3 e_{22}^{2} e_{12}+e_{11}^{2} e_{12}+e_{22}^{2} e_{21}, \\
Q_{124} \equiv & e_{11}^{3} e_{21}+e_{11} e_{22}^{2} e_{21}+e_{11}^{2} e_{22} e_{12}+e_{22}^{3} e_{12}+e_{11} e_{12}^{2} e_{21} \\
& +e_{11} e_{21}^{3}+e_{12}^{3} e_{22}+e_{21}^{2} e_{22} e_{12}
\end{aligned}
$$

Expressions (25)-(37) are exact. However, the quantities $Q_{114}, Q_{224}, Q_{124}$ are of fourth order in $e_{i j}$ and will turn out to be negligible for the purposes of the present study.

We now reduce Cauchy's equation to its simplified form. We first note that it will be convenient to convert the system of second-order (in time) equations (15) into an equivalent system of first-order equations. To do this we will define the particle velocities

$$
v_{i} \equiv \frac{\partial u_{i}}{\partial t},
$$

where $v_{3}=0$ due to the fact that $u_{3}=0$. If we now combine the condition of two-dimensional motions (21), the definition (38), and Cauchy's equation (15), we find that (15) can be rewritten in the following vector form: 


$$
\frac{\partial \mathbf{w}}{\partial t}+\underset{=}{A} \frac{\partial \mathbf{w}}{\partial X_{1}}+\underline{\underline{B}} \frac{\partial \mathbf{w}}{\partial X_{2}}=\mathbf{0},
$$

where $\mathbf{w}$ is a $6 \times 1$ solution vector defined as

$$
\mathbf{w} \equiv\left(\begin{array}{l}
e_{11} \\
e_{12} \\
e_{21} \\
e_{22} \\
v_{1} \\
v_{2}
\end{array}\right)
$$

Here, we note that the last two equations in (39) are the two-dimensional form of Cauchy's equation and the first four arise from the compatibility conditions

$$
\frac{\partial e_{i j}}{\partial t}=\frac{\partial v_{i}}{\partial X_{j}}
$$

obtained by combining the definition (38) with the time differential of (23). The elements of the $6 \times 6$ coefficient matrices $\underset{\underline{A}}{A}$ and $\underline{\underline{B}}$ were found to be

$$
\begin{aligned}
A_{15}= & A_{36}=-1, \\
A_{51}= & -\frac{1}{\rho_{0}}\left[\left(1+e_{22}\right) \frac{\partial \sigma_{11}}{\partial e_{11}}-e_{12} \frac{\partial \sigma_{21}}{\partial e_{11}}\right], \\
A_{53}= & -\frac{1}{\rho_{0}}\left[\left(1+e_{22}\right) \frac{\partial \sigma_{11}}{\partial e_{21}}-e_{12} \frac{\partial \sigma_{21}}{\partial e_{21}}\right], \\
A_{61}= & -\frac{1}{\rho_{0}}\left[\left(1+e_{22}\right) \frac{\partial \sigma_{12}}{\partial e_{11}}-e_{12} \frac{\partial \sigma_{22}}{\partial e_{11}}\right], \\
A_{63}= & -\frac{1}{\rho_{0}}\left[\left(1+e_{22}\right) \frac{\partial \sigma_{12}}{\partial e_{21}}-e_{12} \frac{\partial \sigma_{22}}{\partial e_{21}}\right], \\
B_{51}= & -\frac{1}{\rho_{0}}\left[\left(1+e_{22}\right) \frac{\partial \sigma_{11}}{\partial e_{12}}-e_{12} \frac{\partial \sigma_{21}}{\partial e_{12}}\right. \\
& \left.+\left(1+e_{11}\right) \frac{\partial \sigma_{22}}{\partial e_{11}}-e_{21} \frac{\partial \sigma_{12}}{\partial e_{11}}\right], \\
& \left.+\left(1+e_{11}\right) \frac{\partial \sigma_{21}}{\partial e_{11}}-e_{21} \frac{\partial \sigma_{11}}{\partial e_{11}}\right], \\
B_{54}= & -\frac{\partial}{\rho_{0}}\left[-e_{21} \frac{\partial \sigma_{11}}{\partial e_{22}}+\left(1+e_{11}\right) \frac{\partial \sigma_{21}}{\partial e_{22}}\right], \\
B_{25}= & B_{46}=-1, \frac{\partial \sigma_{12}}{\rho_{0}}-e_{12} \frac{\partial \sigma_{22}}{\partial e_{12}} \\
B_{52}= & -\frac{\partial \sigma_{21}}{\rho_{0}}\left[-e_{21} \frac{\partial \sigma_{11}}{\partial e_{12}}+\left(1+e_{11}\right) \frac{\partial \sigma_{21}}{\partial e_{12}}\right], \\
& \left.+e_{12} \frac{\partial \sigma_{21}}{\partial e_{22}}\right],
\end{aligned}
$$

$$
\begin{aligned}
B_{62} \equiv & -\frac{1}{\rho_{0}}\left[-e_{21} \frac{\partial \sigma_{12}}{\partial e_{12}}+\left(1+e_{11}\right) \frac{\partial \sigma_{22}}{\partial e_{12}}\right], \\
B_{63} \equiv & -\frac{1}{\rho_{0}}\left[\left(1+e_{22}\right) \frac{\partial \sigma_{12}}{\partial e_{22}}-e_{12} \frac{\partial \sigma_{22}}{\partial e_{22}}\right. \\
& \left.+\left(1+e_{11}\right) \frac{\partial \sigma_{22}}{\partial e_{21}}-e_{21} \frac{\partial \sigma_{12}}{\partial e_{21}}\right], \\
B_{64}= & -\frac{1}{\rho_{0}}\left[-e_{21} \frac{\partial \sigma_{12}}{\partial e_{22}}+\left(1+e_{11}\right) \frac{\partial \sigma_{22}}{\partial e_{22}}\right],
\end{aligned}
$$

with all other elements equal to zero. Because the material is isotropic we would naturally expect that the $\underset{=}{A}$ and $\underset{B}{B}$ coefficient matrices would be very similar to each other. However, we have anticipated the fact that we will consider only quasiplane waves which propagate primarily in the $X_{1}$ direction. Most of our calculations will then involve the $\underset{=}{A}$ matrix and we have simplified this matrix by using the definition (23) to replace

$$
\frac{\partial e_{12}}{\partial X_{1}} \text { by } \frac{\partial e_{11}}{\partial X_{2}} \text { and } \frac{\partial e_{22}}{\partial X_{1}} \text { by } \frac{\partial e_{21}}{\partial X_{2}},
$$

wherever they occur.

The undisturbed state of the solid will be taken to be initially at rest and to have at most a small constant shear strain. As a result, we will take the undisturbed state to be

$$
v_{i}=0, \quad u_{1}=u_{3}=0, \quad u_{2}=e_{21}^{(0)} X_{1},
$$

for all $X_{i}$. Here, $e_{21}^{(0)}$ will be taken to be small and constant. The value of the vector (40) in the undisturbed configuration may therefore be written

$$
\mathbf{w}_{0}=\left(\begin{array}{c}
0 \\
0 \\
e_{21}^{(0)} \\
0 \\
0 \\
0
\end{array}\right) .
$$

The main goal of this paper is to derive the evolution equation governing weakly nonlinear, quasi-plane waves. It is well known that longitudinal (or $p$-) waves will have nonnegligible quadratic nonlinearity and will therefore be governed by the classical Khokhlov-Zabolotskaya equation. On the other hand, shear (or $s^{-}$) waves will have zero quadratic nonlinearity in the absence of prestrain. Because the shear mode will be governed by the nonclassical form of the Khokhlov-Zabolotskaya equation described by Kluwick and Cox (1998) and Cramer and Webb (1998), we will focus on the shear mode only.

The wave evolution will be governed by a form of the Khokhlov-Zabolotskaya equation whenever the effects of nonlinearity and diffraction occur at the same rate. For the shear mode this will require that the initial pulse shape should be such that the length scale associated with the transverse variation, i.e., the variations in the $X_{2}$ direction, is of order $L / \epsilon$, where $\epsilon$ is a small, nondimensional parameter measuring the amplitude of the pulse and $L$ is the disturbance length scale in the $X_{1}$ (or propagation) direction. As dis- 
cussed by Kluwick and Cox (1998) and Cramer and Webb (1998), these waves will undergo significant distortion over time scales of order $L /\left(\epsilon^{2} c_{s}\right)$, where $c_{s}$ is the linearized shear wave speed defined more precisely in the next section.

One of the contributions of the present study is to extend the work of Zabolotskaya (1986) and Andrews (1999) to include the effects of prestrain. Here, we will take the prestrain to be small and, in particular, we will take $e_{21}^{(0)}$ $=0(\epsilon)$. With this restriction on the prestrain, the two constraints on the matrices $\underline{\underline{A}}$ and $\underline{\underline{B}}$ which were imposed by Kluwick and Cox (1998) and Cramer and Webb (1998) are satisfied, at least to the accuracy required here. The first constraint requires that the appropriate measure of the quadratic nonlinearity be of order $\epsilon$ at most; the fact that this constraint is satisfied is discussed explicitly in Sec. III of the present study. The second constraint is recorded as Eq. (8) of Cramer and Webb (1998) and ensures that the primary propagation direction is in the $X_{1}$ direction. Although not discussed explicitly in the present paper, it was shown that the appropriate quantity is of $0\left(\epsilon^{2}\right)$ whenever $e_{12}^{(0)}=0(\epsilon)$, and therefore is sufficiently small to apply the theory of Cramer and Webb (1998).

\section{THE MODIFIED KZ EQUATION}

In this section we present the specific form of the modified $\mathrm{KZ}$ equation corresponding to the hyperelastic solid described in Sec. II. Detailed expressions for the solution vector (40) will also be provided. The general form of (39) and the restrictions on the relative sizes of the amplitude and the length and time scales are identical to those considered by Cramer and Webb (1998). Thus, we can simply evaluate the expressions developed by Cramer and Webb (1998) for the specific system of interest here. Because we will frequently need to refer to the article by Cramer and Webb (1998), we will use the shorthand notation $\mathrm{CW}$ for that study.

We should point out that the notation used here will differ slightly from that found in CW. For example, the spatial variables $x$ and $y$ and the dependent variable $\mathbf{u}$ introduced in Eq. (2) of CW will need to be replaced by $X_{1}, X_{2}$, and w, respectively, in the present study.

Under the conditions described in Sec. II of the present investigation and, equivalently, $\mathrm{CW}$, the general solution for (40) is

$$
\mathbf{w}=\mathbf{w}_{0}+\epsilon \mathbf{r} W+\epsilon^{2}\left(\boldsymbol{\delta} V+\boldsymbol{\gamma} \frac{W^{2}}{2}+\mathbf{r} h\right)+0\left(\epsilon^{3}\right),
$$

where $W \equiv W\left(X, X_{2}, t\right)$ is a scalar shape function related to the main disturbance, $V \equiv V\left(X, X_{2}, t\right)$ is a second scalar function related to the disturbances induced by diffraction, and $X \equiv X_{1}-\lambda t$ is a wave coordinate measured in a frame moving at the linear plane-wave speed $\lambda$. The $6 \times 1$ vectors $\mathbf{r}, \boldsymbol{\delta}$, and $\gamma$ provide the relative sizes of the components of $\mathbf{w}$ for the various perturbations of the solution vector $\mathbf{w}$. The quantity $\mathbf{r} h$ seen in (58) represents an arbitrary homogeneous solution parallel to $\mathbf{r}$.

As discussed by $\mathrm{CW}, \mathbf{r}$ and $\lambda$ are the eigenvectors and eigenvalues of $\underline{A}^{(0)} \equiv A\left(\mathbf{w}_{0}\right)=$ speed matrix $\underset{\underline{A}}{ }$ evaluated at the undisturbed state. As in the zero prestrain case studied by
Andrews (1999), a total of six eigenvalues were found for the $A^{(0)}$ matrix associated with (42)-(55). Two of these eigenvalues were zero and correspond to nonpropagating modes. The squares of the eigenvalues corresponding to the propagating modes were found to be

$$
\begin{aligned}
\lambda_{s}^{2}= & c_{s o}^{2}+\frac{e_{21}^{(0) 2}}{2 \rho_{0}}\left\{\left.\frac{\partial^{3} \sigma_{12}}{\partial e_{21}^{3}}\right|_{0}\right. \\
& \left.+\left.\left.\frac{2}{\rho_{0}\left(c_{s o}^{2}-c_{p o}^{2}\right)} \frac{\partial^{2} \sigma_{11}}{\partial e_{21}^{2}}\right|_{0} \frac{\partial^{2} \sigma_{12}}{\partial e_{11} \partial e_{21}}\right|_{0}\right\}+0\left(e_{21}^{(0) 3}\right), \\
\lambda_{p}^{2}= & c_{p o}^{2}+\frac{e_{21}^{(0) 2}}{2 \rho_{0}}\left\{\left.\frac{\partial^{3} \sigma_{11}}{\partial e_{11} \partial e_{21}^{2}}\right|_{0}\right. \\
& \left.+\left.\left.\frac{2}{\rho_{0}\left(c_{p o}^{2}-c_{s o}^{2}\right)} \frac{\partial^{2} \sigma_{11}}{\partial e_{21}^{2}}\right|_{0} \frac{\partial^{2} \sigma_{12}}{\partial e_{11} \partial e_{21}}\right|_{0}\right\}+0\left(e_{21}^{(0) 3}\right),
\end{aligned}
$$

where

$$
\begin{aligned}
& c_{p o} \equiv\left(\left.\frac{1}{\rho_{0}} \frac{\partial \sigma_{11}}{\partial e_{11}}\right|_{0}\right)^{1 / 2}=\left(2 \frac{\tilde{\beta}_{0}+\left.\tilde{\alpha}_{1}\right|_{0}}{\rho_{0}}\right)^{1 / 2}, \\
& c_{s o} \equiv\left(\left.\frac{1}{\rho_{0}} \frac{\partial \sigma_{12}}{\partial e_{21}}\right|_{0}\right)^{1 / 2}=\left(\frac{\left.\tilde{\alpha}_{1}\right|_{0}}{\rho_{0}}\right)^{1 / 2}
\end{aligned}
$$

are the $p$ - and $s$-mode wave speeds in the unstrained state. When we recall (16) the speeds (61)-(62) are recognized as the classical results for the body-wave speeds in linear elasticity.

In the course of the derivation of (59) and (60) it was necessary to show that

$$
\begin{aligned}
& \left.\frac{\partial \tilde{\alpha}_{0}}{\partial e_{11}}\right|_{0}=2 \tilde{\beta}_{0}, \\
& \left.\frac{\partial \tilde{\alpha}_{0}}{\partial e_{21}}\right|_{0}=\left.\frac{\partial \tilde{\alpha}_{1}}{\partial e_{21}}\right|_{0}=\left.\frac{\partial \widetilde{\alpha}_{2}}{\partial e_{21}}\right|_{0}=0, \\
& \left.\frac{\partial^{2} \widetilde{\alpha}_{0}}{\partial e_{21} \partial e_{11}}\right|_{0}=\left.\frac{\partial^{2} \widetilde{\alpha}_{1}}{\partial e_{21} \partial e_{11}}\right|_{0}=\left.\frac{\partial^{2} \widetilde{\alpha}_{2}}{\partial e_{21} \partial e_{11}}\right|_{0}=0 .
\end{aligned}
$$

These results as well as others not recorded here will be used without explicit comment in the remainder of this paper. Examples of the details of such calculations can be found in the thesis of Andrews (1999).

In all that follows we will focus on the shear or s-mode only. When this is done we find that the components of the eigenvector $\mathbf{r}$ corresponding to (59) are

$$
\begin{aligned}
& r_{1}=\left.r_{3} \frac{e_{21}^{(0)}}{\rho_{0}\left(c_{s o}^{2}-c_{p o}^{2}\right)} \frac{\partial^{2} \sigma_{11}}{\partial e_{21}^{2}}\right|_{0}+0\left(e_{21}^{(0) 2}\right), \\
& r_{2}=r_{4}=0
\end{aligned}
$$




$$
r_{5}=-\lambda_{s} r_{1}=-\left.\lambda_{s} r_{3} \frac{e_{21}^{(0)}}{\rho_{0}\left(c_{s o}^{2}-c_{p o}^{2}\right)} \frac{\partial^{2} \sigma_{11}}{\partial e_{21}^{2}}\right|_{0}+0\left(e_{21}^{(0) 2}\right),
$$

$$
r_{6}=-\lambda_{s} r_{3},
$$

respectively. Throughout this paper, we will take $r_{3}$ to be the nonzero but otherwise arbitrary component of the eigenvector $\mathbf{r}$. Following the procedure of $\mathrm{CW}$, we find that the vectors $\boldsymbol{\delta}$ and $\boldsymbol{\gamma}$ can be written

$$
\boldsymbol{\delta}=r_{3}\left(\begin{array}{c}
-1 \\
0 \\
0 \\
1 \\
\lambda_{s} \\
0
\end{array}\right)+0(\epsilon),
$$

and

$$
\boldsymbol{\gamma}=\gamma_{1}\left(\begin{array}{c}
1 \\
0 \\
0 \\
0 \\
-\lambda_{s} \\
0
\end{array}\right)+0(\epsilon)
$$

where

$$
\left.\gamma_{1} \equiv \frac{r_{3}^{2}}{\rho_{0}\left(c_{s o}^{2}-c_{p o}^{2}\right)} \frac{\partial^{2} \sigma_{11}}{\partial e_{21}^{2}}\right|_{0}+0(\epsilon) .
$$

In (67)-(68) any portions of the vectors $\boldsymbol{\delta}$ and $\boldsymbol{\gamma}$ which are parallel to $\mathbf{r}$ can be shown to make no contribution to the resultant evolution equations and, in (58), can be absorbed in the $\mathbf{r} h$ term. Thus, any part of (67)-(68) which is parallel to $\mathbf{r}$ can be ignored with no loss in generality.

An explicit form of the solution for $\mathbf{w}$ can be obtained by combining (58), (63)-(69) with (40) to obtain

$$
\begin{aligned}
& e_{11}=\epsilon r_{1} W+\epsilon^{2}\left[-r_{3} V+\gamma_{1} \frac{W^{2}}{2}\right]+0\left(\epsilon^{3}\right), \\
& e_{12}=0\left(\epsilon^{3}\right), \\
& e_{21}=e_{21}^{(0)}+\epsilon r_{3} W+0\left(\epsilon^{3}\right), \\
& e_{22}=\epsilon^{2} r_{3} V+0\left(\epsilon^{3}\right), \\
& v_{1}=-\lambda_{s} e_{11}+0\left(\epsilon^{3}\right), \\
& v_{2}=-\lambda_{s} e_{21}+0\left(\epsilon^{3}\right) .
\end{aligned}
$$

When we recall that we are taking $e_{21}^{(0)}=0(\epsilon)$, we see that both $e_{11}$ and $e_{22}$ are of second order in the shear pulse amplitude. If we combine (23) and (38) with (74), we can show that the disturbance to $e_{21}$ gives rise to a disturbance in the longitudinal displacement $u_{1}$ which, to lowest order, travels with the main pulse at speed $\lambda_{s}$. The contributions to $e_{11}$ are from three sources. The first contribution can be written as

$$
\begin{aligned}
\epsilon r_{1} W & \left.\approx\left(e_{21}-e_{21}^{(0)}\right) \frac{e_{21}^{(0)}}{\rho_{0}\left(c_{s o}^{2}-c_{p o}^{2}\right)} \frac{\partial^{2} \sigma_{11}}{\partial e_{21}^{2}}\right|_{0} \\
& \approx\left(e_{21}-e_{21}^{(0)}\right) e_{21}^{(0)} \frac{\gamma_{1}}{r_{3}^{2}}
\end{aligned}
$$

where we have used (63), (69), and (72). This term represents the part of $e_{11}$ generated by the interaction of the shear wave and the prestrain. The second contribution $-\epsilon^{2} r_{3} V$ $\approx-e_{22}$ is due to the diffraction, i.e., the transverse variations. The final contribution is due to the nonlinearity of the stress-strain relation (5) and can be written

$$
\epsilon^{2} \gamma_{1} \frac{W^{2}}{2} \approx \frac{\gamma_{1}}{2 r_{3}^{2}}\left(e_{21}-e_{21}^{(0)}\right)^{2}
$$

where we have used (72).

The fact that the passage of the shear wave causes a density perturbation of second order can be seen by adding (70) to (73). We then use (63), (69), and (72) to obtain

$$
e_{11}+e_{22} \approx \frac{1}{2} \frac{\gamma_{1}}{r_{3}^{2}}\left(e_{21}^{2}-e_{21}^{(0) 2}\right)+0\left(\epsilon^{3}\right) .
$$

If we further expand (14) and the last of (24) in a manner which is consistent with (70)-(75), we find that

$$
\frac{\rho}{\rho_{0}} \approx 1-\frac{1}{2}(I I I-1)+0\left(\epsilon^{4}\right) \approx 1-\left(e_{11}+e_{22}\right)+0\left(\epsilon^{4}\right),
$$

which, when combined with (76), yields

$$
\frac{\rho}{\rho_{0}} \approx 1-\frac{1}{2} \frac{\gamma_{1}}{r_{3}^{2}}\left(e_{21}^{2}-e_{21}^{(0) 2}\right)+0\left(\epsilon^{3}\right),
$$

where $\gamma_{1} / r_{3}^{2}$ can be obtained from (69). Result (77) is consistent with the well-known result that material nonlinearity can generate density perturbations under shear loads.

We now turn to the determination of the specific form of the $\mathrm{KZ}$ equation corresponding to the hyperelastic material described in Sec. II. In CW the general form of the KZ equation in terms of the shape functions $W$ and $V$ was given. If we use (72) and (73) to replace $W$ and $V$ by $e_{21}$ and $e_{22}$, we find that the evolution equations governing weakly nonlinear, weakly diffracting waves in hyperelastic solids are

$$
\begin{aligned}
& \frac{\partial e_{21}}{\partial t} \pm c_{s o}\left[\widetilde{\Gamma}+\frac{\tilde{\Lambda}}{2}\left(e_{21}-e_{21}^{(0)}\right)\right]\left(e_{21}-e_{21}^{(0)}\right) \frac{\partial e_{21}}{\partial X} \pm \frac{c_{s o}}{2} \frac{\partial e_{22}}{\partial X_{2}} \\
& \quad=0 \\
& \frac{\partial e_{22}}{\partial X}=\frac{\partial e_{21}}{\partial X_{2}},
\end{aligned}
$$

to lowest order. Here, $X \equiv X_{1}-\lambda_{s} t$ 


$$
\begin{aligned}
\widetilde{\Lambda} \equiv & \frac{1}{2 \rho_{0} c_{s o}^{2}}\left[\left.\frac{\partial^{3} \sigma_{12}}{\partial e_{21}^{3}}\right|_{0}\right. \\
& \left.+\left.\left.\frac{3}{\rho_{0}\left(c_{s o}^{2}-c_{p o}^{2}\right)} \frac{\partial^{2} \sigma_{11}}{\partial e_{21}^{2}}\right|_{0} \frac{\partial^{2} \sigma_{12}}{\partial e_{21} \partial e_{11}}\right|_{0}\right]_{0}+0(\epsilon), \quad(80) \\
\widetilde{\Gamma} \equiv & \frac{e_{21}^{(0)}}{2 \rho_{0} c_{s o}^{2}}\left[\left.\frac{\partial^{3} \sigma_{12}}{\partial e_{21}^{3}}\right|_{0}+\left.\frac{3}{\rho_{0}\left(c_{s o}^{2}-c_{p o}^{2}\right)} \frac{\partial^{2} \sigma_{11}}{\partial e_{21}^{2}} \frac{\partial^{2} \sigma_{12}}{\partial e_{21} \partial e_{11}}\right|_{0}\right] \\
& +0\left(e_{21}^{(0) 2}\right) \approx e_{21}^{(0)} \tilde{\Lambda} .
\end{aligned}
$$

The \pm signs in (78) correspond to right and left running waves, and the differential with respect to time seen in (78) is taken holding $X$ and $X_{2}$ constant.

Once (78)-(79) are solved for $e_{21}$ and $e_{22}, e_{11}$ can be determined from (76) and $u_{1}, u_{2}$ can be determined by straightforward integration once it is recognized that (71) implies that $u_{1}=u_{1}\left(X_{1}, t\right)$ to lowest order.

We note that (79) can be further simplified through use of (23) and conversion back to $X_{1}, X_{2}, t$ coordinates to yield

$$
\frac{\partial^{2} u_{2}}{\partial X_{1} \partial X_{2}}=\frac{\partial^{2} u_{2}}{\partial X_{2} \partial X_{1}} .
$$

Thus, (79) can be recognized as a compatibility condition on the shear displacement $u_{2}\left(X_{1}, X_{2}, t\right)$.

The term proportional to $\widetilde{\Gamma}$ introduces quadratic nonlinearity into the evolution equation (78). Inspection of (81) reveals that $\widetilde{\Gamma} \rightarrow 0$ as the prestrain $e_{21}^{(0)} \rightarrow 0$. Thus, our restriction that $e_{21}^{(0)}=O(\epsilon)$ guarantees that the quadratic nonlinearity is weak and therefore that condition (12) of CW holds.

The term proportional to $\widetilde{\Lambda}$ in (78) represents cubic nonlinearity and is seen to be consistent with that obtained by Zabolotskaya (1986) for transverse waves in a nonlinear isotropic solid once differences in coordinate systems are taken into account. We also note that the one-dimensional version of (78) is consistent with the work of Carman and Cramer (1992) when their result is simplified using the small disturbance approximation.

In the course of the derivation of (78)-(79), it was found that the terms $A, B$ defined in Eqs. (28) and (29) of CW were of order $\epsilon$ and are therefore negligible in the physical system considered here. The coefficients $A$ and $B$ are proportional to the coefficients $\beta^{\prime \prime \prime}$ and $\beta^{(i v)}$ in (4) of the present investigation and represent terms generated by an interaction of the weak nonlinearity and the weak diffraction. Thus, in spite of the complexity of the original physical system (39)-(40) and (42) - (55), the primary effect of the small prestrain is seen to be the modification of the linear wave speed (59) and the addition of the term representing quadratic nonlinearity to the KZ equations of Zabolotskaya (1986) and Andrews (1999).

Because the scaled value of the quadratic nonlinearity coefficient $\widetilde{\Gamma}$ is proportional to $\widetilde{\Lambda}$, it can be shown that we can recast (78) in an alternate form which reads

$$
\frac{\partial e_{21}}{\partial t} \pm c_{s o} \frac{\tilde{\Lambda}}{2}\left(e_{21}^{2}-e_{21}^{(0) 2}\right) \frac{\partial e_{21}}{\partial X} \pm \frac{c_{s o}}{2} \frac{\partial e_{22}}{\partial X_{2}}=0 .
$$

This form of the evolution equation is particularly useful when considering the effect of the sign of the prestrain $e_{21}^{(0)}$ on the wave motion. Inspection of (83) reveals that the evolution is invariant with respect to the sign of $e_{21}^{(0)}$. This conclusion may not be immediately obvious when we recall that the quadratic nonlinearity parameter (81) changes sign as the prestrain changes sign.

Finally, we note that the evolution equation (78) can be reduced to a modified or cubic $\mathrm{KZ}$ equation by differentiation with respect to $X$ and use of (79); the result is found to be

$$
\left[e_{t} \pm c_{s o}\left(\widetilde{\Gamma} e+\frac{\tilde{\Lambda}}{2} e^{2}\right) e_{X}\right]_{X} \pm \frac{c_{s o}}{2} e_{X_{2} X_{2}}=0
$$

where we have replaced $e_{21}-e_{21}^{(0)}$ by $e$ for convenience and the subscripts $X, X_{2}, t$ again denote partial derivatives. Equation (84) is of the same general form as (3) with an additional term representing quadratic nonlinearity.

A second reduction of (78)-(79) to a single equation can be obtained by noting that (79) implies the existence of a potential function. In fact, (82) implies that the appropriate potential is the shear displacement $u_{2}$. It will be convenient to define

$$
u \equiv u_{2}-e_{21}^{(0)} X_{1}
$$

which is a measure of the actual shear displacement from the undisturbed state. When (85) is combined with (23) and (78), we find that

$$
u_{X t} \pm c_{s o}\left[\tilde{\Gamma} u_{X}+\frac{\tilde{\Lambda}}{2}\left(u_{X}\right)^{2}\right] u_{X X} \pm \frac{c_{s o}}{2} u_{X_{2} X_{2}}=0,
$$

where we have used the fact that $e_{22}=u_{X_{2}}$ and

$$
e_{21} \equiv \frac{\partial u_{2}}{\partial X_{1}}=\frac{\partial u_{2}}{\partial X} \approx \frac{\partial u}{\partial X}+e_{21}^{(0)}
$$

We note that (86) is a cubically nonlinear version of the low-frequency transonic equation derived by Cramer and Seebass (1978) in the context of focusing shock waves in perfect gases.

In closing this section we note that Eqs. (78)-(79), (84), and (86) are written in a coordinate system which translates at speed $\lambda_{s}$ in the $X_{1}$ direction. When we transform back to a frame which is stationary with respect to the undisturbed solid, we find that the counterpart of (78)-(79) can be obtained simply by replacing $X\left(\equiv X_{1}-\lambda_{x} t\right), X_{2}, t$ by $X_{1}, X_{2}$, $t$ in (78)-(79) and by adding the term $\lambda_{s} \partial e_{21} / \partial X_{1}$ to the result of replacing $X, X_{2}, t$ by $X_{1}, X_{2}, t$. As a final step, it can also be shown that no additional terms arise when our equations are converted from the Lagrangian coordinates $X_{i}$ to the Eulerian coordinates $x_{i}$. That is, it was found that the $\mathrm{KZ}$ equation was essentially the same form regardless of whether Lagrangian or Eulerian coordinates are employed, at least to the accuracy needed here. 


\section{DISSIPATIVE WAVES}

In this section we show how weak dissipation modifies the evolution equation (78). Simple dissipation will be included through use of a Kelvin-Voigt model. To incorporate this model we write the stress (5) as

$$
\sigma_{i j}=\sigma_{i j}^{(e)}+\sigma_{i j}^{(v)},
$$

where $\sigma_{i j}^{(e)}$ is the elastic part of the stress identical to (5). The second term is the viscous part of the stress given by

$$
\sigma_{i j}^{(v)} \equiv \lambda_{v} \frac{\partial v_{\ell}}{\partial X_{\ell}} \delta_{i j}+\mu_{v}\left(\frac{\partial v_{i}}{\partial X_{j}}+\frac{\partial v_{j}}{\partial X_{i}}\right),
$$

where $\mu_{v}$ and $\lambda_{v}$ are shear and second viscosities, respectively. As discussed by $\mathrm{CW}$, only the lowest-order contributions to the dissipative terms will be required. As a result, the linear stress-rate of strain relation (88) will be adequate for our purposes. Furthermore, $\mu_{v}$ and $\lambda_{v}$ may be taken to be constants and the distinction between Lagrangian and Eulerian coordinates can be ignored in (88).

When (87)-(88) are substituted in (15), we find that the system of equations governing the wave propagation is

$$
\begin{aligned}
\frac{\partial \mathbf{w}}{\partial t}+\underline{\underline{A}} \frac{\partial \mathbf{w}}{\partial X_{1}}+\underline{\underline{B} \frac{\partial \mathbf{w}}{\partial X_{2}} \approx} & \stackrel{\underline{D}}{(x x)} \frac{\partial^{2} \mathbf{w}}{\partial X_{1}^{2}}+\underline{\underline{D}}^{(x y)} \frac{\partial^{2} \mathbf{w}}{\partial X_{1} \partial X_{2}} \\
& +\underline{\underline{D}}^{(y y)} \frac{\partial^{2} \mathbf{w}}{\partial X_{2}^{2}},
\end{aligned}
$$

where $\mathbf{w}, \mathbf{w}_{0}, \underline{\underline{A}}, \underline{\underline{B}}$ are identical to those given in Sec. II. The only difference between (89) and (39) is the presence of the dissipative terms having the $N \times N$ matrices $\underline{\underline{D}}^{(x x)}, \underline{\underline{D}}^{(x y)}$, $\underline{D}^{(y y)}$ as coefficients. The only nonzero components of the latter are found to be

$$
\begin{aligned}
& D_{55}^{(x x)}=\frac{\lambda_{v}+2 \mu_{v}}{\rho_{0}}, \quad D_{66}^{(x x)}=\frac{\mu_{v}}{\rho_{0}}, \\
& D_{56}^{(x y)}=D_{65}^{(x y)}=\frac{\lambda_{v}+\mu_{v}}{\rho_{0}}, \\
& D_{55}^{(y y)}=\frac{\mu_{v}}{\rho_{0}}, \quad D_{66}^{(y y)}=\frac{\lambda_{v}+2 \mu_{v}}{\rho_{0}} .
\end{aligned}
$$

The effects of weak dissipation on the shear waves will become noticeable at times of order $\rho_{0} L^{2} / \mu_{v}$. If the dissipative effects are much larger than the effects of nonlinearity and diffraction, the shear pulse will damp out long before the latter effects will be noticeable. On the other hand, pulses for which the dissipation is much weaker than the nonlinearity will evolve with no noticeable dissipation, except in the interior of shock waves, over the time scales considered here. We will therefore take the length scale, $L$, and the pulse amplitude to be such that dissipation, nonlinearity, and diffraction all occur at the same rate. As a result, we require that $\rho_{0} L^{2} / \mu_{v}=0\left(L /\left(\epsilon^{2} c_{s o}\right)\right)$, or that

$$
\operatorname{Re}_{s} \equiv \frac{c_{s o} \rho_{0} L}{\mu_{v}}=0\left(\frac{1}{\epsilon^{2}}\right),
$$

where $\operatorname{Re}_{s}$ is recognized as the Reynolds number based on the linearized shear wave speed.

An extension of the Khokhlov-Zabolotskaya equation to include weak relaxation, dissipation, and dispersion has been derived by $\mathrm{CW}$ for the case where these effects are of the same strength as the nonlinearity and diffraction. When Cramer and Webb's scheme is applied to (89), it is found that the dissipative form of the evolution equation (78) may now be written

$$
\begin{aligned}
\frac{\partial e_{21}}{\partial t} & \pm c_{s o}\left[\widetilde{\Gamma}+\frac{\tilde{\Lambda}}{2}\left(e_{21}-e_{21}^{(0)}\right)\right]\left(e_{21}-e_{21}^{(0)}\right) \frac{\partial e_{21}}{\partial X} \pm \frac{c_{s o}}{2} \frac{\partial e_{22}}{\partial X_{2}} \\
& =\frac{\mu_{v}}{2 \rho_{0}} \frac{\partial^{2} e_{21}}{\partial X^{2}},
\end{aligned}
$$

with (79) unchanged. We note further that the inclusion of material dissipation leaves the basic solutions (70)-(75) unchanged. As a result, the procedure for finding $u_{1}$ and $u_{2}$ from $e_{21}, e_{22}$ is exactly the same as in the nondissipative case. The only difference between the dissipative and nondissipative cases is the difference in the governing equations (78) and (94). The evolution equation (94) is again seen to be completely consistent with previous investigations as well as the dispersion relation for linear Kelvin-Voigt solids.

Because (79) is unchanged when dissipation is included, we can immediately write down the extensions of (83), (84), and (86); these read

$$
\begin{aligned}
& \frac{\partial e_{21}}{\partial t} \pm c_{s o} \frac{\tilde{\Lambda}}{2}\left(e_{21}^{2}-e_{21}^{(0) 2}\right) \frac{\partial e_{21}}{\partial X} \pm \frac{c_{s o}}{2} \frac{\partial e_{22}}{\partial X_{2}}=\frac{\mu_{v}}{2 \rho_{0}} \frac{\partial^{2} e_{21}}{\partial X^{2}}, \\
& {\left[e_{t} \pm c_{s o}\left(\widetilde{\Gamma} e+\frac{\tilde{\Lambda}}{2} e^{2}\right) e_{X}\right]_{X} \pm \frac{c_{s o}}{2} e_{X_{2} X_{2}}=\frac{\mu_{v}}{2 \rho_{0}} e_{X X X},}
\end{aligned}
$$

and

$$
u_{X t} \pm c_{s o}\left[\tilde{\Gamma} u_{X}+\frac{\tilde{\Lambda}}{2}\left(u_{X}\right)^{2}\right] u_{X X} \pm \frac{c_{s o}}{2} u_{X_{2} X_{2}}=\frac{\mu_{v}}{2 \rho_{0}} u_{X X X},
$$

where $e$ and $u$ have the same definitions as in Sec. III and the subscripts $X, X_{2}, t$ in (96)-(97) denote partial differentiation.

\section{SHOCK JUMP CONDITIONS}

It is expected that the presence of nonlinearity and dissipation will generally give rise to the formation of shock waves. The shock jump relations can be derived in a standard way by first converting (78)-(79) to a conservative form and then by integrating over a volume enclosing the possible discontinuity. In terms of the original $X_{1}, X_{2}, t$ system, we find that the jump conditions for a right-running shock wave can be written

$$
\begin{aligned}
& \frac{s}{\lambda_{s}} \approx 1+\frac{\tilde{\Gamma}}{2} \frac{\llbracket e^{2} \rrbracket}{\llbracket e \rrbracket}+\frac{\tilde{\Lambda}}{6} \frac{\llbracket e^{3} \rrbracket}{\llbracket e \rrbracket}+o\left(\epsilon^{2}\right), \\
& \llbracket e_{22} \rrbracket \approx \theta_{s} \llbracket e \rrbracket+o\left(\epsilon^{2}\right),
\end{aligned}
$$

where $e$ is again simply $e_{21}-e_{21}^{(0)}$, the double brackets denote the jump in the indicated quantity across the shock, and 
$\theta_{s}=0(\epsilon)$ is the acute angle between the shock surface and the positive $X_{2}$ axis, measured in a counterclockwise direction. The quantity $s$ is the dimensional shock speed relative to the original $X_{1}, X_{2}, t$ frame.

We note that the lowest-order shock speed (98) is identical to that derived in the one-dimensional theory of Carman and Cramer (1992). From the point of view of the shock, the primary way in which the two-dimensional character of the motion is incorporated is through the jump condition (99).

An important feature of shock waves in nonlinear solids can be revealed by an inspection of the characteristic surfaces associated with (78)-(79). If the equation of the characteristic surface is $F\left(X_{1}, X_{2}, t\right)=0$, then the unit normal (n) and speed $\left(s_{c}\right)$ of this surface can be written

$$
\mathbf{n} \equiv \frac{\nabla F}{|\nabla F|}, \quad s_{c} \equiv-\frac{F_{t}}{|\nabla F|},
$$

respectively. If we apply the method of characteristics to (78)-(79) and recognize that $n_{X_{2}}=0\left(\epsilon n_{X_{1}}\right)=o\left(n_{X_{1}}\right)$, we find that the speed of a right-running characteristic surface can be approximated by

$$
\frac{s_{c}}{\lambda_{s}}=1+\widetilde{\Gamma} e+\frac{\widetilde{\Lambda}}{2} e^{2}+o\left(\epsilon^{2}\right) .
$$

Thus, the speed of the characteristic surface is only dependent on $e \equiv e_{21}-e_{21}^{(0)}$ and is identical to that of the onedimensional theory described by Carman and Cramer (1992).

We close this section by noting that the necessary and sufficient conditions for the local existence of shear shocks can be written

$$
s_{c 1} \leqslant s \leqslant s_{c 2} ;
$$

that is, the shock speed is required to be larger than the wave speed immediately ahead of the shock (defined as $s_{c 1}$ ) and smaller than the wave speed immediately behind the shock (defined as $s_{c 2}$ ). Condition (102) is recognized as Lax's (1971) speed-ordering condition and holds in a trivial way even for cases having strictly quadratic nonlinearity. The derivation of (102) is based either on an analysis of the dissipative structure of the shock or on the basis of a casualty argument, i.e., an argument denying the possiblity that events in the present or past can be influenced by events which occur in the future.

A graphical interpretation of (102) can be developed by defining the flux function

$$
Q=Q(e) \equiv \frac{\widetilde{\Gamma}}{2} e^{2}+\frac{\widetilde{\Lambda}}{6} e^{3} .
$$

A sketch of the $Q$ vs $e$ curve for $\widetilde{\Gamma}>0, \widetilde{\Lambda}>0$ has been provided in Fig. 1. Straightforward differentiation can be used to show that

$$
Q^{\prime}=\widetilde{\Gamma} e+\frac{\widetilde{\Lambda}}{2} e^{2} \approx \frac{s_{c}}{\lambda_{s}}-1,
$$

where $Q^{\prime} \equiv d Q / d e$. Futhermore

$$
\frac{\llbracket Q \rrbracket}{\llbracket e \rrbracket}=\frac{\widetilde{\Gamma}}{2} \frac{\llbracket e^{2} \rrbracket}{\llbracket e \rrbracket}+\frac{\tilde{\Lambda}}{6} \frac{\llbracket e^{3} \rrbracket}{\llbracket e \rrbracket}=\frac{s}{\lambda_{s}}-1 .
$$

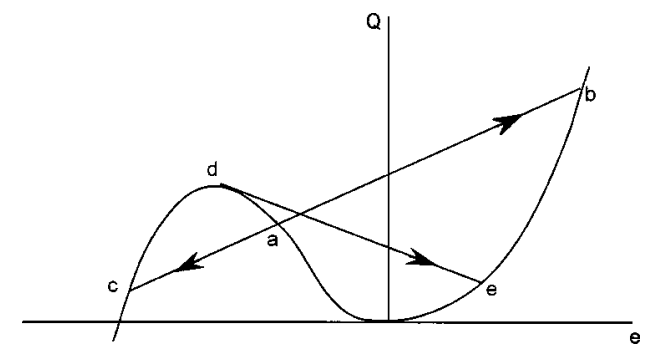

FIG. 1. Sketch of the flux function (103) for the case $\widetilde{\Gamma}, \widetilde{\Lambda}>0$. Arrows denote the direction of jumps for admissible shocks and d-e denotes a sonic shock.

Thus, the slope of the $Q$ vs $e$ curve is closely related to the speed of the characteristic surfaces, and the slope of the straight line connecting the states ahead of and behind the shock is closely related to the shock speed. Furthermore, (102) can be recast as

$$
Q_{1}^{\prime} \leqslant \frac{\llbracket Q \rrbracket}{\llbracket e \rrbracket} \leqslant Q_{2}^{\prime},
$$

where $Q_{i}^{\prime} \equiv Q^{\prime}\left(e_{i}\right)$ for $i=1,2$. As in (102), the subscripts 1 and 2 will denote states ahead of and behind the shock, respectively. Thus, the admissibility of a proposed shear discontinuity can be determined by a relatively simple inspection of the corresponding $Q$ vs $e$ curve. As an example, we consider the states $a, b, c$ indicated on the $Q$ curve in Fig. 1 . The states $a$ and $b$ will correspond to states on either side of an admissible shock, i.e., one satisfying (102) or (106), if and only if $a$ is the state ahead of the shock and $b$ is the state behind the shear shock. To emphasize this fact we have added an arrow in Fig. 1 to indicate the direction of the admissible jump. If we consider a discontinuity between the points $a$ and $c$ in Fig. 1, we can conclude that the only admissible shock is one for which the solid is taken from state $a$ to state $c$. Thus, both $e$-lowering and $e$-raising shocks are possible if both $\tilde{\Lambda}$ and $\widetilde{\Gamma}$ are positive; it can be shown that the same conclusion will be valid if $\widetilde{\Lambda}$ and $\widetilde{\Gamma}$ are simply nonzero.

An example of an inadmissible discontinuity is one having end states given by $c$ and $b$. Because the slopes of the $Q$ curve at $c$ and $b$ are greater than the slope of the chord $c-b$, (106) will always be violated for this discontintuity.

Tangency points are also clearly possible. One such shock is represented by the states $d-e$ in Fig. 1. Such tangency points correspond to the equalities in (102) and (106) and represent a shear shock for which the shock speed is identical to the characteristic speed immediately ahead of or behind the shock. The resultant shock wave is referred to as a sonic shock and is not possible in the classical KZ theory. A detailed analysis of the $Q$ curve in Fig. 1 shows that the sonic shocks will correspond to the maximum or minimum shock strength when such an extremum is not zero or infinite. Furthermore, experience with the one-dimensional theory strongly suggests that sonic shocks will be the result of partial disintegrations of inadmissible discontinuities.

Finally, we consider the unstrained undisturbed state discussed by Zabolotskaya (1986). In this case $e_{21}^{(0)}=0, \widetilde{\Gamma}=0$, and $e=e_{21}$. Thus, (103) reduces to the pure cubic relation 


$$
Q=\frac{\tilde{\Lambda}}{6} e^{3} .
$$

Sonic shocks as well as shocks carrying both increases and decreases of $e$ are possible. An interesting special case is that where $\widetilde{\Lambda}<0$. In this case, no shocks are possible which have $e=0$ ahead of the shock. Thus, when the prestrain is zero, a disturbance propagating into an undisturbed media, i.e., one having $e=0$, cannot be headed by a shock regardless of the degree of focusing or attenuation.

\section{RESULTS FOR A BLATZ-KO MATERIAL}

In this section we consider a specific material model and provide explicit formulas for the nonlinearity coefficients derived in Sec. III. The model chosen is that developed by Blatz and Ko (1962) for compressible foam rubbers. To specify any particular material we simply need to give the dependence of the strain energy on the invariants $I, I I, I I I$. For the Blatz-Ko model this relation is

$$
\begin{aligned}
U= & \frac{\mu_{0} b}{2 \rho_{0}}\left\{I-3+\frac{1-2 \nu}{\nu}\left(I I I^{-\nu /(1-2 \nu)}-1\right)\right\} \\
& +\frac{\mu_{0}(1-b)}{2 \rho_{0}}\left\{\frac{I I}{I I I}-3+\frac{1-2 \nu}{\nu}\left(I I I^{\nu /(1-2 \nu)}-1\right)\right\},
\end{aligned}
$$

where $\mu_{0}, \nu$, and $b$ are material constants.

It can be shown that the Lamé constants (16) for the Blatz-Ko material can be written

$$
\begin{aligned}
& \left.\mu^{\prime} \equiv \tilde{\alpha}_{1}\right|_{0}=\mu_{0}, \\
& \lambda^{\prime} \equiv 2 \widetilde{\beta}_{0}=2 \mu_{0} \frac{\nu}{1-2 \nu} .
\end{aligned}
$$

Thus, the Blatz-Ko parameters $\mu_{0}$ and $\nu$ are just the shear modulus and Poisson ratios of the linear theory. As a result, these parameters may be determined by examining the material response in the small strain limit. The parameter $b$ cannot be determined in the limit of linear elasticity. Blatz and Ko (1962) performed experiments showing that $\mu_{0}$ $\approx 32 \mathrm{psi} \approx 221 \mathrm{kPa}, b \approx 0$, and $\nu=1 / 4$ for the foamed polyurethene rubbers generated in their lab. Further discussion of the Blatz-Ko model and its limitations can be found in the original article and those by Beatty and Stalnaker (1986) and Beatty (1987).

When (108) is substituted in (8)-(10), we find that

$$
\begin{aligned}
& \alpha_{0}=\mu_{0}\left\{(1-b)\left[I I I^{(4 \nu-1) /[2(1-2 \nu)]}-\frac{I I}{I I I^{3 / 2}}\right]\right. \\
& \left.-b I I I^{-1 /[2(1-2 \nu)]}\right\}, \\
& \alpha_{1}=\frac{\mu_{0}}{I I I^{1 / 2}}\left\{b+\frac{I}{I I I}(1-b)\right\}, \\
& \alpha_{2}=-\mu_{0} \frac{1-b}{I I I^{3 / 2}},
\end{aligned}
$$

exactly. We note in passing that the values of $\alpha_{0}$ and $\alpha_{1}$ given by Carman and Cramer (1992) contained typographical errors in the authors' original manuscript. However, those typographical errors had no effect on their reported results.

Results (111)-(113) may now be combined to yield the specific forms of (17)-(19); these read

$$
\begin{aligned}
\tilde{\alpha}_{0}= & \mu_{0}(1-b)\left[I I I^{(4 \nu-1) /[2(1-2 v)]}+\frac{I-I I-1}{I I I^{3 / 2}}\right] \\
& +\mu_{0} b\left[I I I^{-1 / 2}-I I I^{-1 /[2(1-2 v)]}\right], \\
\tilde{\alpha}_{1}= & \mu_{0}\left[\frac{b}{I I I^{1 / 2}}+(1-b) \frac{I-2}{I I I^{3 / 2}}\right], \\
\tilde{\alpha}_{2}= & \alpha_{2}=-\mu_{0} \frac{1-b}{I I I^{3 / 2}},
\end{aligned}
$$

exactly. Results (114)-(115) were used to generate the expressions for the Lamé constants (109)-(110) for the Blatz-Ko material. The results for $\widetilde{\beta}_{0},\left.\widetilde{\alpha}_{1}\right|_{0}$ may be combined with (61)-(62) to obtain the linearized shear and longitudinal wave speeds for the unstrained material. In the remainder of this section we use (114)-(116) to compute the parameters associated with the nonlinear aspects of the wave propagation.

We begin by noting that the only derivatives of $\sigma_{i j}$ which are required can be written

$$
\begin{aligned}
& \left.\frac{\partial^{2} \sigma_{11}}{\partial e_{21}^{2}}\right|_{0}=2\left[\left.\frac{\partial \widetilde{\alpha}_{0}}{\partial I}\right|_{0}+\left.\frac{\partial \widetilde{\alpha}_{0}}{\partial I I}\right|_{0}+\left.\tilde{\alpha}_{2}\right|_{0}\right], \\
& \left.\frac{\partial^{2} \sigma_{12}}{\partial e_{21} \partial e_{11}}\right|_{0}=2 \widetilde{\beta}_{1}+\left.\widetilde{\alpha}_{1}\right|_{0}+\left.2 \tilde{\alpha}_{2}\right|_{0}, \\
& \left.\frac{\partial^{3} \sigma_{12}}{\partial e_{21}^{3}}\right|_{0}=6\left[\left.\frac{\partial \tilde{\alpha}_{1}}{\partial I}\right|_{0}+\left.\frac{\partial \widetilde{\alpha}_{1}}{\partial I I}\right|_{0}+\left.\tilde{\alpha}_{2}\right|_{0}\right],
\end{aligned}
$$

where

$$
\left.\widetilde{\beta}_{1} \equiv \frac{\partial \widetilde{\alpha}_{1}}{\partial I}\right|_{0}+\left.2 \frac{\partial \widetilde{\alpha}_{1}}{\partial I I}\right|_{0}+\left.\frac{\partial \tilde{\alpha}_{1}}{\partial I I I}\right|_{0} .
$$

Details of the derivations leading to (117)-(120) can be found in the thesis by Andrews (1999). When (114)-(116) are substituted in (117)-(120), we find that

$$
\begin{aligned}
& \left.\frac{\partial^{2} \sigma_{11}}{\partial e_{21}^{2}}\right|_{0}=\left.\frac{\partial^{2} \sigma_{12}}{\partial e_{21} \partial e_{11}}\right|_{0}=-2 \mu_{0}(1-b), \\
& \left.\frac{\partial^{3} \sigma_{12}}{\partial e_{21}^{3}}\right|_{0}=0 .
\end{aligned}
$$

We may now determine the parameters (69) and (80)-(81) for the Blatz-Ko material. The lowest-order values of these quantities read

$$
\frac{\gamma_{1}}{r_{3}^{2}}=\frac{2 c_{s o}^{2}}{c_{p o}^{2}-c_{s o}^{2}}(1-b),
$$




$$
\tilde{\Lambda}=-\frac{6 c_{s o}^{2}}{c_{p o}^{2}-c_{s o}^{2}}(1-b)^{2}, \quad \widetilde{\Gamma}=\tilde{\Lambda} e_{21}^{(0)} .
$$

In like manner, the perturbation to the linear wave speed and density may be computed by substituting (121)-(123) in (59) and (77) to yield

$$
\begin{aligned}
& \lambda_{s} \approx \pm c_{s o}\left[1-e_{21}^{(0) 2} \frac{2 c_{s o}^{2}}{c_{p o}^{2}-c_{s o}^{2}}(1-b)^{2}+0\left(\epsilon^{3}\right)\right], \\
& \frac{\rho}{\rho_{0}}=1-\frac{c_{s o}^{2}}{c_{p o}^{2}-c_{s o}^{2}}(1-b)\left[e_{21}^{2}-e_{21}^{(0) 2}\right]+0\left(\epsilon^{3}\right) .
\end{aligned}
$$

If we make the usual assumptions that $\mu^{\prime}>0$ and $0<\nu<1 / 2$ for any linear material, then $c_{p o}^{2}>c_{s o}^{2}$. Thus, (124)-(125) may be used to show that $\tilde{\Lambda}<0$ and that the magnitude of the linear wave speed is decreased by any nonzero prestrain $e_{21}^{(0)}$. If we further take $b<1$ we conclude that shear waves decrease the material density from $\rho_{0}$ whenever $\left|e_{21}\right|$ $>\left|e_{21}^{(0)}\right|$. In particular, $\rho<\rho_{0}$ for a $b<1$ Blatz-Ko material having no prestrain, at least in the context of the small disturbance theory presented here.

Inspection of (123)-(126) reveals that the effects of nonlinearity are related in a simple way to the material constant $b$. Blatz and Ko (1962) found that $b \approx 0, \nu=1 / 4$ for their polyurethene foams, whereas they found that $b \approx 1, \nu \approx 0.463$ for solid polyurethene rubber. If we note that

$$
\frac{c_{s o}^{2}}{c_{p o}^{2}-c_{s o}^{2}}=1-2 \nu,
$$

we find that

$$
\frac{\gamma_{1}}{r_{3}^{2}} \approx 1, \quad \tilde{\Lambda} \approx-3, \quad \tilde{\Gamma} \approx-3 e_{21}^{(0)},
$$

for Blatz and Ko's polyurethene forms. On the other hand, the nonlinearity appears to vanish in the limit of $b \rightarrow 1$. That is, the Blatz-Ko model predicts that the propagation of quasi-plane shear waves is at most quartic in the $b \rightarrow 1$ limit. Experience has shown that the evolution equation must be modified even when $1-b=0(\epsilon)$. Under these conditions, the time scale over which nonlinearity becomes noticeable is proportional to $\epsilon^{-3}$ rather than the $\epsilon^{-2}$ used here. Furthermore, diffraction can balance the weakened nonlinearity only if the transverse gradients are also correspondingly weaker.

Such a reduction in the nonlinearity should not be surprising when we note that (108) reduces to

$$
U \approx \frac{\mu_{0}}{2 \rho_{0}}(I-3),
$$

when $b \approx 1$; here, we have noted that (126) implies that the density perturbations induced by the shear wave are of order $\epsilon^{3}$ at most. The strain energy (127) is recognized as the energy of a neo-Hookean solid which in turn is just a simplification of the classical Mooney-Rivlin strain enegy. It is well known that the Mooney-Rivlin model yields a linear response in a simple (or homogeneous) shear.
It should be noted that other, more accurate, models of solid rubbers have nonzero cubic nonlinearity parameters. One such example is Ogden's (1972) model. The behavior of the nonlinearity coefficients as $b \rightarrow 1$ seen here should be regarded as a characteristic of the Blatz-Ko and MooneyRivlin family of models rather that as a general statement of the behavior of all solid rubbers.

\section{SUMMARY}

We have examined weakly nonlinear, weakly diffracting shear waves in a hyperelastic solid. The extension of the Khokhlov-Zabolotskaya equation which is valid for small shear prestrains has been derived. Two alternate forms of our extended $\mathrm{KZ}$ equation were presented; the nondissipative forms are given by (78)-(79), (84), and (86) and the dissipative forms are given by (95)-(97).

The primary effect of the prestrain was seen to be to modify the linear wave speed and to change the nonlinearity from a pure cubic nonlinearity to a mixed cubic and quadratic nonlinearity. Surprisingly, the terms representing interactions between nonlinearity and diffraction found in the general modified $\mathrm{KZ}$ equation derived by Kluwick and Cox (1998) and CW were found to be negligible even when the prestrain is nonzero. To the authors' knowledge, the present case is the largest and most complicated physical system for which such nonlinear diffraction terms play no role in the lowest order evolution.

A second new result is that for the shock jump conditions (98)-(99). The shock existence conditions were also given in terms of the speed-ordering condition (102). We have also provided a short discussion of a graphical approach to the analysis of the admissibility of proposed discontinuities. A new type of shock, not possible in the classical KZ theory and referred to as a sonic shock, was briefly described. Experience with the one-dimensional theory suggests that such shocks will play a key role in the nonlinear diffraction of shear shocks.

Finally, we have given examples of the nonlinearity constants for a specific material model. The model chosen was based on the well-known Blatz-Ko formula (108) for the strain energy. The explicit form of the relevant constants is given by (124). If we take $\mu^{\prime}>0,0<\nu<1 / 2$, and $b \neq 1$, (124) reveals that $\widetilde{\Lambda}<0$ for all Blatz-Ko materials. If we further restrict attention to the case of no prestrain studied by Zabolotskaya (1986), a disturbance propagating into an undisturbed solid will never be headed by a shock wave. In this $e_{12}^{(0)}=0, \widetilde{\Lambda}<0$ case, the resultant wavefront will always be smooth and will travel with the linearized wave speed.

Andrews, M. F. (1999). "Evolution equations for weakly nonlinear, quasiplanar waves in isotropic dielectrics and elastomers," Masters thesis, Virginia Polytechnic Institute and State University, Blacksburg, Virginia.

Beatty, M. F. (1987). "Topics in finite elasticity: Hyperelasticity of rubber, elastomers, and biological tissues_with examples," Appl. Mech. Rev. 40(12), 1699-1734.

Beatty, M. F., and Stalnaker, D. O. (1986). "The Poisson function of finite elasticity," J. Appl. Mech. 53, 807-813.

Blatz, P. J., and Ko, W. L. (1962). "Application of finite elastic theory to the deformation of rubbery materials," Trans. Soc. Rheol. VI, 223-251. 
Carman, G. P., and Cramer, M. S. (1992). "Nonlinearity parameters for pulse propagation in isotropic elastomers," J. Acoust. Soc. Am. 91(1), 39-51.

Cramer, M. S., and Seebass, A. R. (1978). "Focusing of weak shock waves at an arête," J. Fluid Mech. 142, 9-37.

Cramer, M. S., and Webb, C. (1998). "A Zabolotskaya-Khokhlov equation for cubic and near-cubic nonlinearity," AIAA Paper AIAA-98-2957.

Kluwick, A., and Cox, E. A. (1998). "Nonlinear waves in materials with mixed nonlinearity," Wave Motion 27, 23-41.

Lax, P. D. (1971). "Shock waves and entropy," in Contributions to Nonlinear Functional Analysis, edited by E. H. Zarantonello (Academic, New York).
Norris, A. N. (1998). "Finite-amplitude waves in solids," in Nonlinear Acoustics, edited by M. F. Hamilton and D. T. Blackstock (Academic, New York), pp. 263-277.

Ogden, R. W. (1972). "Large deformation isotropic elasticity-on the correlation of theory and experiment for incompressible rubberlike solids," Proc. R. Soc. London, Ser. A 326, 565-584.

Zabolotskaya, E. A. (1986). "Sound beams in a nonlinear isotropic solid," Sov. Phys. Acoust. 32(4), 296-299.

Zabolotskaya, E. A., and Khokhlov, R. V. (1969). "Quasi-plane waves in the nonlinear acoustics of confined beams," Sov. Phys. Acoust. 15(1), $35-40$. 